\title{
Development and Implementation of Pacific Wide Telehealth Services in Gastroenterology
}

\author{
Christopher S. HAIR ${ }^{\mathrm{a}, 1}$ Mai Ling PERMAN ${ }^{\mathrm{b}}$ Payne PERMAN ${ }^{\mathrm{c}}$ Joji MALANI $^{\mathrm{b}}$ \\ Vikash SHARMA ${ }^{\mathrm{b}}$ Aminiasi ROKOCAKAU ${ }^{\mathrm{b}}$ Dimitri ORLEANSKI $^{\mathrm{d}}$ \\ Jerry KENA ${ }^{\mathrm{d}}$ Folototo LEAVAI ${ }^{\mathrm{e}}$ Miguel BROSTROM ${ }^{\mathrm{f}}$ and Christina HIGA ${ }^{\mathrm{f}}$ \\ ${ }^{a}$ Department of Medicine, University Hospital Geelong, Australia \\ ${ }^{b}$ College of Medicine, Nursing and Health Sciences, Fiji National University, Fiji \\ ${ }^{c}$ Pohnpei State Hospital, Pohnpei, Federated States of Micronesia \\ ${ }^{d}$ Department of Medicine, LBJ Tropical Medical Center, American Samoa \\ ${ }^{e}$ Department of Medicine, Tupua Tamasese Meaole Hospital, Samoa \\ ${ }^{f}$ Pacific Basin Telehealth Resource Center, Social Science Research Institute, \\ University of Hawai 'i, USA
}

\begin{abstract}
Gastroenterology, as a sub-speciality in medicine, has been widely developed across the Pacific Nations during the last decade as a result of 'hub' training in Suva, Fiji. Professional isolation for trained pacific gastroenterologists has remained an issue due to the high cost of travel, limited in-country workforce, and recently COVID-19 border closures. Whereas Telehealth solutions have been used for decades across many Pacific Island Nations as a means to improve the clinical outcomes in Pacific Island patients, only recently has this technology been cleverly adopted in fostering education and skill development in Pacific gastroenterology. In this paper, we describe the evolution of these various methods and discuss their use and adaptation in various clinical situations. Herein we consider the future of telehealth programs and the potential for positive impact on Pacific Island healthcare.
\end{abstract}

Keywords: Distance learning, telehealth, Pacific Island Nations, gastroenterology, endoscopy, instant messaging

\section{Introduction}

The Oceanic Pacific Region better known as Oceania has a vast land area of over 8 million square kilometres and is home to a population of over 40 million people (1). Oceania incorporates the developed nations of Australia and New Zealand as well as the Pacific Island Nations, with their three main groups Melanesia, Micronesia and Polynesia. These 22 island nations and territories are home to 11 million people and are bordered by vast areas of ocean (2). Due to the vast distances between nations and small populations per island nation, there are significant challenges in accessing and providing healthcare within these small countries $(2,3)$.

1 Corresponding author: Christopher S Hair, Epworth Private Hospital, 1 Epworth Place, Waurn Ponds, Victoria, Australia; Email: chris@drchrishair.com 
Gastroenterology illnesses are highly prevalent in these regions, including several malignancies and infectious enteric illnesses $(4,5,6)$. Historically, the opportunity for an early diagnosis and treatment of gastrointestinal disease in the pacific islands has been related to limited access to trained professionals, a limited capacity to endoscopy and other diagnostic procedures, as well as the high costs of travel for patients to a referral hospital $(7,8,9,10)$.

In 2008, a centralised 'hub' based gastroenterology and endoscopy training program was developed at the Colonial War Memorial Hospital (CWMH), Suva, Fiji (10). Regional Pacific Island doctors and nurses can undertake specialist training at this centre under the supervision of local and international visiting gastroenterologists, endoscopists and nurses. Over this period of time there have been more than 200 attendances from local and regional doctors and nurses at the centre, many attending for training on more than 1 occasion. This program is celebrated as one of the most successful pacific programs in upgrading technical skills, building capacity and improving knowledge in gastroenterology for Fiji and the Pacific Island Countries. The training centre conducts its program in collaboration with the Australian and New Zealand Gastroenterology International Training Association (ANZGITA) and the World Gastroenterology Organisation (WGO).

As a result of this successful collaboration, there has been wide distribution of skilled gastroenterology clinicians and nurses across the Pacific Island Nations, who now provide access to endoscopy and gastroenterology treatments for Pacific Island people in countries including Timor Leste, Solomon Islands, Federated States of Micronesia (Pohnpei and Chuuk), Marshall Islands, Palau, Vanuatu, Fiji, Samoa, Kiribati, Tuvalu, American Samoa and Tonga. However, once returned to these countries, these upskilled healthcare providers have remained challenged by many factors including professional isolation $(11,12)$. This isolation has included limited access to timely specialist mentor support during clinic appointments and with acutely unwell hospitalised patients, as well as mentor support during elective and emergency endoscopy. Furthermore, there has been an expressed desire to remain up-to-date with pacific relevant gastroenterology knowledge and guidelines.

In order to limit the impact of isolation, several telehealth solutions have been developed and implemented since 2017. The goals of these solutions have been to provide ongoing mentoring and support of pacific island healthcare practitioners in gastroenterology and endoscopy when required. These methods have been locally developed in collaboration with international partnerships and have included monthly case discussions and webinars, instant messaging solutions for complex pacific cases, real-time tele-endoscopy mentoring and case based learning using 'celli-medicine' technology. The overarching principles of these solutions meet the expressed need of Pacific community including curriculum that is sensitive to the local needs, culture and level of technology (13).

\section{Development, format and Implementation of Pacific Gastroenterology telehealth seminars}

\subsection{Pacific Gastroenterology Telehealth Seminar}

The Pacific Gastroenterology Telehealth Seminar (PGTS) was first proposed by the authors as a potential solution to professional isolation in education and case support in 
2017. During this time there had been a rapid, successful development in novel telehealth solutions in Pohnpei, Micronesia including tele-echocardiography and tele-pathology (14). There were pre-existing global examples of clinical support programs, including Project ECHO, which the authors drew elements from as a model for the PGTS (15). Project ECHO is a novel tele-medicine based strategy based around the 'hub-and-spoke' design and increases knowledge sharing by multiplying expert knowledge beyond a oneone teaching or didactic model and amplifies learning through an all-teach-all-learn model $(16,17)$.

Building on the success of Project ECHO, the PGTS elements include a brief presentation on a gastroenterology focused topic by an international specialist group, followed by a Pacific-wide clinical discussion around the topic, followed by opportunity to discuss relevant or emerging cases. Developing and moderating a group discussion around emerging cases was particularly relevant, as many healthcare problems in the Pacific require a unique understanding of the local capacity to manage the disease as well as a knowledge of disease presentation, which is often different to that seen in the West.

It was considered by the authors that the PGTS could be made widely accessible to healthcare specialists from across the Pacific Nations using pre-existing networks. Much of these networks were already in place as a consequence of the successful work from the Pacific Basin Telehealth Resource Center (PBTRC) Hawaii (18) and through the CWMH Fiji Hub program with ANZGITA (10). The PGTS is made available using Zoom video conferencing facilitated by the host (PBTRC). Invitation to clinicians is widely distributed through email, through instant messenger networks (Viber/messenger) and local healthcare networks via the co-ordinated networks of ANZGITA and the South Pacific Community (SPC) health care subgroup.

Since the inaugural session in 2017, a monthly PGTS has been conducted. The program format includes a welcome and recognition of each attendee, a 15-minute presentation on a gastrointestinal (GI) topic, followed by a group discussion moderated by a central facilitator. Since inception, 25 webinars have been conducted with a broad reaching regular audience including 20-30 individuals from clinical teams from American Samoa, Samoa, Tonga, Fiji, Marshall Islands, Chuuk, Pohnpei, Palau, Kosrae, Yap, Guam, Vanuatu, Solomon Islands, Australia, New Zealand, Hawaii and Canada.

The curriculum for presentation is regularly discussed and planned with the authors of this paper, to ensure that presentations remain focussed on the needs of the Pacific Island healthcare professionals. The small group nature of the PGTS facilitates discussion between healthcare practitioners, and allows for meaningful practical outcomes to clinical cases discussed. Furthermore, the context provides a safe, friendly and open platform for discussions so that healthcare practitioners feel welcomed and valued to express their opinions which enhances the discussions.

\subsection{Connecting small networks by instant messenger app to promote case based learning}

Whilst the PGTS provides a regular, scheduled monthly gathering to discuss and learn from a wider group of clinicians, prior to 2017 there was no capacity for fast trans-Pacific discussions and education on interesting or relevant cases. In recognition of the successes of the discussion network of the PGTS, we developed the Pacific Internal Medicine Grand Round (PIMGR) using instant messenger facilitation. By 2017, most Pacific Clinicians had access to a mobile device, and would regularly use Viber ${ }^{\mathrm{TM}}$ as a method to communicate within their healthcare network. 
We postulated the creation of a huge Viber clinical network, where clinicians from the PGTS would join and post in discussion, would facilitate a regular e-learning network. The PIMGR became the primary platform for a central discussion site on challenging or interesting cases in Internal Medicine including peptic ulcer disease presentations through to Fish Poisoning and pulmonary disorders.

Unlike the PGTS, where a scheduled and planned educational session was conducted, the PIMGR took advantage of an opportunistic moment in medicine, such as at the time of clinical contact with a patient, or during a challenging GI procedure. Since inception, the PIMGR has had over 50 case posts and discussions, most of which have assisted the clinical outcome of the patient.

Due to the success of the PIMGR, there have been several sub-groups developed in order to discuss even more relevant or specific disease processes. These have included an endoscopy focused subgroup in the Solomon Islands, a gastroenterology subgroup incorporating Pohnpei, Fiji and Australia, and a surgical subgroup incorporating Fiji and Australia surgeons.

\subsection{Use of telemedicine in emergency endoscopy, Samoa}

Samoa is a remote Polynesian island nation with a population of approximately 200,000 . Endoscopy re-emerged in Samoa during 2016 following a brief period of use in the late 1990s. The initial experience of endoscopy in Samoa was short-lived due to a lack of ongoing support from abroad and limited capacity to perform the procedure on island (19).

Several trained endoscopists (surgical and medical) and endoscopy nurses have returned from the Suva hub training in Fiji to work in general medicine, general surgery and nursing since 2015 and they have recommenced regular endoscopy procedures on a weekly basis as well as lifesaving emergency endoscopy in urgent cases. Subsequent training for these health professionals has been carried out in country on an annual basis since 2017 by members of ANZGITA. Over time there is a gradual evolution of clinical competency, including disease recognition as well as theatre capacity through equipment support and maintenance. However, a need for a more regular mentorship, particularly pathology recognition and disease management has existed.

Within the endoscopy suite at Tupua Tamaese Meaole Hospital, Samoa, there is no access to fast internet. This prohibits the capacity to truly perform 'live streamed endoscopy'. To overcome the barrier of isolation during endoscopy, Samoan clinicians have become accustomed to using their own mobile devices installed with instant messenger apps (mainly Viber) to send video, photo and case reports to mentor endoscopy specialists in Australia, New Zealand and Fiji. On receipt of this message the specialist has been instantly able to advise on diagnosis and management options, mostly whilst the procedure is being performed in Samoa. In most cases, this has led to a change in management thinking or a different strategy for negotiating a skill difficulty.

More recently, this strategy had the spectacular result of saving the life of a Samoan patient with life-threatening bleeding from the stomach. The attending Samoan team had recognised the bleeding pathology but required assistance in performing a skill through the endoscope that would control the bleeding point. Using Viber, a series of live videos were recorded, sent to and remotely accessed by an Australian specialist, who was able to provide near-real time live guidance and advice during the performance of the lifesaving measure. The patient made a successful recovery without complication. Without this tele-mentoring approach, this patient would have died from this condition. Since this 
event, the concept of using an instant messenger assisted endoscopy has grown to be used in more cases in Samoa, Palau, American Samoa, Fiji, Vanuatu and the Solomon Islands.

\subsection{Initial experience with tele-endoscopy mentoring, Pohnpei, Federated States of Micronesia}

There is a developing global interest in providing tele-mentorship to isolated clinicians in surgical procedures. Tele-mentoring has been recently studied in various laparoscopic surgeries and urological procedures with early positive outcome data, and tele-mentoring has been previously reviewed in upper airway endoscopy $(20,21,22)$. Unlike surgical procedures, the e-mentoring or tele-mentoring during gastrointestinal endoscopy has rarely been utilized and is only now in infantile stages of development and safety assessment. Of particular relevance, and as noted in the Samoa example, many Pacific Island clinicians who perform endoscopy are challenged by recognition of pathology, or assistance during a procedure to a new skill or technology.

Our initial experience with tele-endoscopy took place in Pohnpei. Third author of Pohnpei State Hospital conducted a complex gastrointestinal endoscopy while a panel of experts from ANZGITA and Fiji School of Medicine offered real time advice and guidance by video teleconference. At the same time, other participants (Kosrae, American Samoa, Palau, Fiji and Japan) who joined the telehealth session learned much by observing and asking questions.

To assure optimal video image and teleconference quality, Pohnpei Hospital used a high-definition camera and a new desktop screen so that the Pacific Island partners could be seen by the Pohnpei team during the procedure. The procedure involved the insertion of an endoscope into the oesophagus and stomach and the use of a balloon dilator that was inserted into the scope and through a pathological narrowing to widen it. This is a specialized procedure that is usually done by experienced endoscopist.

Third author was able to receive real time mentoring by the experts who observed and gave advice along the way. Experts were able to advise on what instruments and sizes of instruments to use as well as help with the next steps in managing this patient. The procedure was safely performed, and the patient demonstrated a good clinical outcome. This avoided the need for expensive transfer of the patient off island for the procedure as well as developed the confidence and skill of the third author. Since the inaugural e-mentoring, Pohnpei has conducted several subsequent elective real-time endoscopy procedures with mentoring provided from Fiji and Australia.

\subsection{Other programs and opportunities}

There are many other examples of telehealth support across the Pacific Island Nations that are noteworthy and valuable to mention. As treatment options for viral hepatitis B begins to be made available across several Pacific Island Nations, there is a need to support health clinicians with treatment dilemmas as well as testing and treatment protocols. A highly valuable zoom-led education and clinical support program in hepatitis B treatment program has been implemented in Tonga, Kiribati and Fiji. This program incorporates education and training modules delivered in real-time online, as well as clinical support at the bedside level.

Since 2009 members of ANZGITA have enjoyed travel to Fiji and conducted a month-long education program in gastroenterology to postgraduate doctors undertaking 
their Master of Medicine or diploma of medicine studies at the Fiji National University (FNU). The program has consisted of four weeks of lectures and tutorials covering aspects of gastroenterology that are relevant in the Pacific region. During the COVID19 pandemic of 2020, global travel and access to Pacific Island Nations had temporarily ceased. Many of the postgraduate clinicians remained in their regional countries in the Pacific but were able to continue their studies remotely. To overcome this barrier, the 2020 gastroenterology program was redesigned and produced collaboratively with FNU academic staff and ANZGITA using Zoom as the platform to conduct several academic meetings between Fiji, Australia and New Zealand. The program was then conducted entirely via Zoom by academic clinicians from Australia and New Zealand, facilitated by FNU, and joined by the postgraduate clinicians from Timor Leste, Vanuatu, Solomon Islands, Tonga, Samoa, and Fiji.

Finally, a number of Pacific conference presentations have been delivered using zoom during the past 3 years including 2 gastroenterology presentations to the Pacific Basin Medical Association (PBMA) annual medical conference in Palau and Pohnpei by ANZGITA volunteer academics who would not have been able to attend in person and deliver these critical keynote addresses.

\section{Limitations and Future Directions}

Whilst many gastroenterology tele-technology solutions that support Pacific clinicians have been widely embraced across the Pacific Island Nations healthcare during the past few years, there is much work that can be done to optimize supportive healthcare.

\subsection{Creating digital equity across Pacific healthcare}

There is a need for digital equity across all healthcare settings. Whilst much of the U.S. affiliated nations including Palau, Pohnpei and Marshall Islands have advanced telehealth settings, access to telehealth support in several nations is in infancy of development, or unavailable. For example, in Port Vila, Vanuatu telehealth is limited to phone and Viber, with limited capacity for live streaming of endoscopy and webinars due to lack of access issues. Only recently, the National Referral Hospital in Honiara, Solomon Islands has come online with access to high-speed internet in its clinical setting. The remote islands of Kiribati and Tuvalu, similarly, have limited access. Many clinicians in larger nations such as Fiji and Samoa are still limited to use of their own mobile devices for e-support in clinical areas despite high-speed internet capabilities on island.

\subsection{Challenges with non-clinical educational professional time}

There are also several other broader challenges that face participating pacific island clinicians including access to non-clinical time to participate in webinar-based discussions. The broad time difference from the most eastern Pacific Island nations to the most western is 5 hours, and this creates scheduling challenges the capacity for teams to attend scheduled sessions. The impact of COVID-19 and other illness outbreaks such as Measles, Samoa (2019) at least temporarily reduces available clinical time and teams to participate in group discussions. In fact, during COVID-19, many island doctors have 
faced the challenge of prioritizing a greater number of 'available' tele-education opportunities in their small amount of non-clinical time allocation.

\subsection{Ethical Issues and risks associated with tele-gastroenterology}

There are a number of important ethical and risk considerations for the clinician participating in gastroenterology tele-medicine. For supportive volunteer clinicians who offer their advice and expertise across nations, there is risk of medical litigation, perhaps even more so during e-mentoring during higher risk clinical procedures such as colonoscopy. Whilst several of these issues are discussed elsewhere (23), there is currently no clear, global guidance or global medico-legal support to these clinicians and in most cases, it remains up to the individual to either accept these risks and seek clarification from their own medical indemnity insurer or decline to participate in the clinical situation.

Despite all of these challenges, there remains great excitement, motivation and clinical need to develop digital technology solutions in support of gastroenterology and endoscopy speciality services beyond 2020 . There are valuable emerging opportunities in education and training of sub-speciality gastroenterology nurses, bio-technicians and allied health clinicians, who all provide significant care to patients with gastroenterology diseases. As Pacific Island Nations face the impact of climate change and deal with the healthcare impacts of COVID-19, there will be a greater need to develop and embrace digital technology that facilitates and enhances the capacity to successfully provide collaboration, collegiate support, education and mentorship. The Pacific Island clinicians and their diverse health services are, in many ways, already ahead of the many other nations in this regard.

\section{References}

[1] World Population prospects - Population division population.un.org United Nations Department of Economic and Social Affairs, Population Division.

[2] The Lancet. Saving the Pacific islands from extinction. Lancet. 2019 Aug 3;394(10196):359.

[3] Sweet L, Young L, Kado S. Enhancing health professional education capacity in the Western Pacific region. Rural Remote Health. 2018 Aug;18(3):4306.

[4] Torgerson PR, Hagan JE, Costa F, Calcagno J, Kane M, Martinez-Silveira MS, Goris MG, Stein C, Ko AI, Abela-Ridder B. Global Burden of Leptospirosis: Estimated in Terms of Disability Adjusted Life Years. PLoS Negl Trop Dis. 2015 Oct 2;9(10):e0004122.

[5] Moore MA, Baumann F, Foliaki S, Goodman MT, Haddock R, Maraka R, Koroivueta J, Roder D, Vinit T, Whippy HJ, Sobue T. Cancer epidemiology in the pacific islands - past, present and future. Asian Pac J Cancer Prev. 2010;11 Suppl 2(0 2):99-106.

[6] Teng AM, Blakely T, Baker MG, Sarfati D. The contribution of Helicobacter pylori to excess gastric cancer in Indigenous and Pacific men: a birth cohort estimate. Gastric Cancer. 2017 Jul;20(4):752-755.

[7] Bunkley N, Bissett I, Buka M, Bong J, Leodoro B, Dare A, Perry W. A Household Survey to Evaluate Access to Surgical Care in Vanuatu. World J Surg. 2020 Oct;44(10):3237-3244.

[8] Negin J, Martiniuk AL, Farrell P, Dalipanda T. Frequency, cost and impact of inter-island referrals in the Solomon Islands. Rural Remote Health. 2012;12:2096. Epub 2012 Sep 19.

[9] Watters DA, Guest GD, Tangi V, Shrime MG, Meara JG. Global Surgery System Strengthening: It Is All About the Right Metrics. Anesth Analg. 2018 Apr;126(4):1329-1339..

[10] https:/www.worldgastroenterology.org/education-and-training/training-centers/centers/wgo-suvatraining-center

[11] Negin J. Australia and New Zealand's contribution to Pacific Island health worker brain drain. Aust N Z J Public Health. 2008 Dec;32(6):507-11.

[12] Watters DA, McCaig E, Nagra S, Kevau I. Surgical training programmes in the South Pacific, Papua New Guinea and Timor Leste. Br J Surg. 2019 Jan;106(2):e53-e61. 
[13] PACNET/Western Pacific HealthNet (WPHNet) Pacific Telehealth Conference (1998, Noumea, New Caledonia) Report of meeting .1. Telecommunication in medicine- Oceania-Congresses I. Secretariat of the Pacific Community II. Pacific Basin Medical Association

[14] Higa C. Exploring Key Elements for Successful Telehealth Implementation Within Severely UnderResourced Communities: A Case Study in the Pacific Islands. Stud Health Technol Inform. 2018;254:2432.

[15] Arora S, Kalishman S, Thornton K, et al. Expanding access to hepatitis C virus treatment-Extension for Community Healthcare Outcomes (ECHO) project: Disruptive innovation in specialty care. Hepatology 2010;52(3):1124-33.

[16] Zhou C, Crawford A, Serhal E, Kurdyak P, Sockalingam S.. The impact of project ECHO on participant and patient outcomes: A systematic review. Acad Med 2016;91(10):1439-61.

[17] Katzman JG, Comerci G, Boyle JF, et al. . Innovative telementoring for pain management: Project ECHO Pain. J Contin Educ Health Prof 2014;34(1):68-75.

[18] Higa C, Okamura J, Okamoto S, Okamura N. Health Information Technology and Telehealth Challenges in the U.S. Affiliated Pacific Islands Region. Stud Health Technol Inform. 2018;254:33-42.

[19] Watson K, Asaua M, Dean M. Upper gastrointestinal endoscopy in Samoa and a changed protocol for peptic ulcer. Pacific health dialog. Mar 1999; v.6 n.1:p35-38

[20] Mizota T, Kurashima Y, Poudel S, Watanabe Y, Shichinohe T, Hirano S. Step-by-step training in basic laparoscopic skills using two-way web conferencing software for remote coaching: A multicenter randomized controlled study. Am J Surg. 2018 Jul;216(1):88-92.

[21] Soucisse ML, Boulva K, Sideris L, Drolet P, Morin M, Dubé P. Video Coaching as an Efficient Teaching Method for Surgical Residents-A Randomized Controlled Trial. J Surg Educ. 2017 Mar-Apr;74(2):365371. doi: 10.1016/j.jsurg.2016.09.002. Epub 2016 Oct 5.

[22] Huang EY, Knight S, Guetter CR, Davis CH, Moller M, Slama E, Crandall M. Telemedicine and telementoring in the surgical specialties: A narrative review. Am J Surg. 2019 Oct;218(4):760-766.

[23] Langarizadeh M, Moghbeli F, Aliabadi A. Application of Ethics for Providing Telemedicine Services and Information Technology. Med Arch. 2017 Oct;71(5):351-355. 